\title{
Comparison of the Time-resolved Absorption and Phosphorescence from the Tryptophan Triplet State in Proteins in Solution
}

\author{
Anne Gershenson*, Ari Gafni and Duncan Steel† \\ The Institute of Gerontology, The University of Michigan, Ann Arbor, MI, USA
}

Received 19 August 1997; accepted 7 January 1998

\begin{abstract}
Measurement of the room temperature Trp triplet state lifetime in proteins by time-resolved phosphorescence can provide valuable information on the structure and dynamics of proteins in solution. Our time-resolved absorption measurements on the long-lived states resulting from electronic excitation of the chromophore demonstrate the presence of more complex behavior than revealed by time-resolved phosphorescence. To provide additional insight into this behavior, a comparative study of time-resolved transient absorption and time-resolved phosphorescence of proteins in solution was carried out. The results show that the time evolution of the long-lived states observed through transient absorption often differs considerably from that observed in time-resolved phosphorescence. In some proteins, the presence of competing reactions complicates the interpretation of the transient absorption measurements (which may affect the phosphorescence yield). A more complete characterization of these processes will likely prove useful in the study of protein structure and dynamics in solution.
\end{abstract}

\section{INTRODUCTION}

Measurements of time-resolved room temperature phosphorescence (RTP) $\ddagger$ emission from tryptophan (Trp) residues of proteins in solution is a relatively novel approach that has only recently been applied to the study of protein structure. The first demonstration of RTP in solution was by Saviotti and Galley on liver alcohol dehydrogenase and Escherichia coli alkaline phosphatase (AP) (1). Their work showed the importance of reducing the concentration of oxygen, a highly effective triplet state quencher, to subnanomolar levels. In the absence of molecular oxygen, many proteins phospho-

*Present address: California Institute of Technology, Chemical Engineering 210-41, Pasadena, CA 91125 , USA.

†To whom correspondence should be addressed at: The Institute of Gerontology, 300 North Ingalls Building, The University of Michigan, Ann Arbor, MI 48109, USA. Fax: 313-936-2116; e-mail: dst@umich.edu

$\$$ Abbreviations: AP, Escherichia coli alkaline phosphatase; apoAz, apoazurin; $\mathrm{e}_{\mathrm{aq}}^{-}$, solvated electron; PMT, photomultiplier tube; rPGK, rabbit muscle phosphoglycerate kinase; RTP, room temperature phosphorescence; $\operatorname{Trp}^{+}$, tryptophan cation radical; Típ, tryptophan neutral radical; yPGK, yeast phosphoglycerate kinase.

(c) 1998 American Society for Photobiology 0031-8655/98 $\$ 5.00+0.00$ resce in solution at ambient temperature (2-4). Numerous studies have shown that the RTP lifetime, which can vary from microseconds to seconds, depends on the degree of exposure to either intrinsic or extrinsic quenchers (3-5). Without such quenching, the RTP lifetime has been shown to depend sensitively on the local rigidity, as inferred from a comparison of RTP lifetime and solvent viscosity $(6,7)$. Thus, small changes in quenching and/or in local rigidity can have a measurable effect on Trp RTP lifetimes (compared, for example, to the small influence of these factors on the fluorescence, which occurs on the nanosecond scale). Due to this sensitivity, time-resolved RTP may be used to detect the subtle conformational changes that accompany various reactions.

For example, quenching of RTP by energy transfer has been used to study the structure of AP (8) and has been suggested as an experimental approach that would enable high spatial resolution through accurate site-to-site distance measurements (9). By following the RTP lifetime as a function of time, it has also been possible to identify transient intermediates during protein unfolding (10) and refolding (11). Room temperature phosphorescence has been used to detect conformational changes that can occur, for example, as a function of $\mathrm{pH}(12,13)$. In addition to studies of the RTP lifetime, RTP contains circularly polarized emission due to the local chiral environment, similar to fluorescence. The circularly polarized component in the phosphorescence emission is an order of magnitude larger than observed in fluorescence and can be time resolved (14) to provide additional information (15). Use of RTP for structural studies has been further enhanced by using protein engineering techniques to introduce or remove Trp residues (16-20). For a review of the methodology see Schauerte et al. (21).

To further our understanding of the triplet state and its sensitivity to structure and to changes in the local environment, we have performed a series of experiments to provide insight into the dynamics of the triplet state and the associated photophysics. The approach is based on our development of a laser-based spectrometer system that allows us to measure time-resolved excited-state absorption associated with the triplet state. As the results below demonstrate, new information is available through this approach, although the interpretation is more complex. More specifically, time-resolved transient absorption measurements on proteins can detect the Trp triplet state as well as dark photochemical 


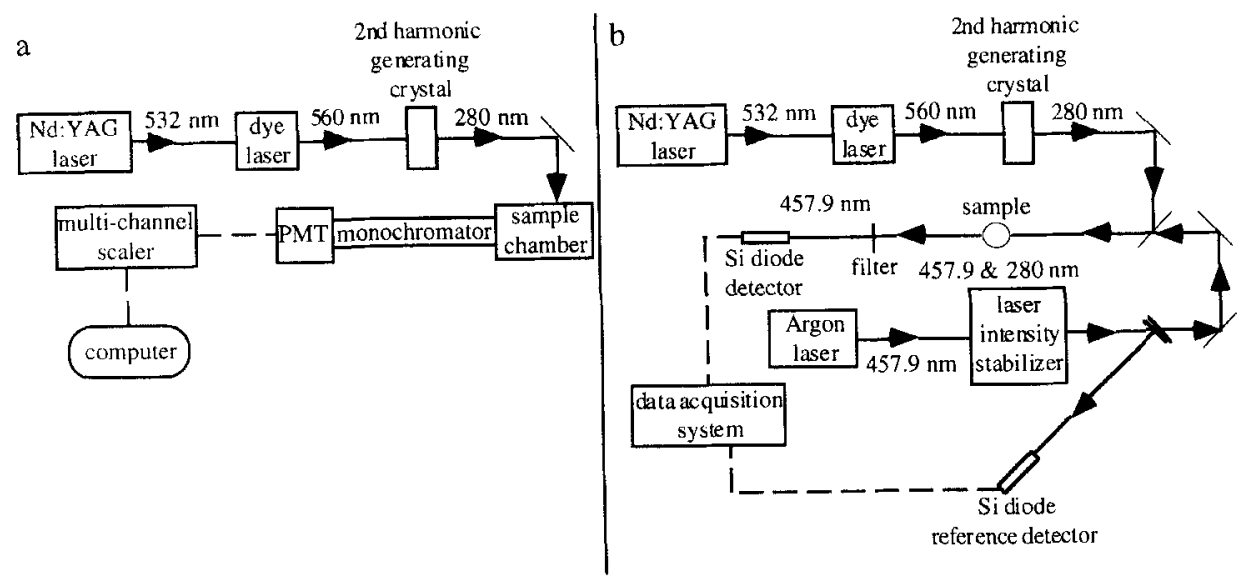

Figure 1. Schematic representation of the instrumentation used to record time-resolved phosphorescence (a) and transient absorption (b). For details see the text.

species such as the Trp cation radical $\left(\operatorname{Trp}^{+}\right)$, the Trp neutral radical (Trp) and the solvated electron $\left(e_{a q}^{-}\right)(22-25)$. The detection of other transiently absorbing species and the determination of their lifetimes can complement RTP measurements because such data contain information about the environment surrounding phosphorescing Trp residues and provide a window into the chemistry that occurs following photoexcitation.

Differentiation of the various contributions to the transient absorption can be partially accomplished by comparing the absorption of an aerated solution with that of a deoxygenated one. In general, triplet states are so rapidly quenched by collisions with oxygen that their absorption will not be detected on time scales of microseconds or longer. The $e_{a q}^{-}$are also rapidly scavenged by molecular oxygen. The transient absorption of free radical species ( $\operatorname{Trp}^{+}$, Trp) is not seriously affected by the presence of oxygen although the signal from these species may be decreased if a significant proportion of the radicals result from photochemistry of the triplet state. With further measurement, additional differentiation can be achieved by resolution of the transient absorption spectra into the various spectral contributions known to arise from each of the different species $(26,27)$.

In order to demonstrate the complimentarity and efficacy of transient absorption measurements comparative data are presented of lifetimes obtained from RTP and transient absorption for AP and Pseudomonas aeruginosa apoazurin (apoAz) as well as for yeast phosphoglycerate kinase (yPGK), which is reportedly phosphorescent at room temperature $(4,28,29)$, and for PGK from rabbit muscle (rPGK).

\section{MATERIALS AND METHODS}

Sample preparation. Escherichia coli AP (type III) was obtained as a suspension in $2.5 \mathrm{M}$ ammonium sulfate from Sigma Chemical Co. (St. Louis, MO). The ApoAz (from the bacterial strain ATCC 19429) was generously provided by Professor Graham Fleming (University of Chicago). Yeast PGK was purchased from Sigma in lyophilized form and from Boehringer Mannheim (Indianapolis, IN) as a suspension in $3.2 \mathrm{M}$ ammonium sulfate. Rabbit PGK was obtained from Sigma in lyophilized form. Protein samples were desalted and concentrated using Amicon (Beverly, MA) Centricons into $50 \mathrm{~m} M$ Tris, $\mathrm{pH} 7.5$ for AP and apoAz, $20 \mathrm{~m} M$ triethanolamine $\mathrm{HCl}, \mathrm{pH} 7.5$ or $20 \mathrm{~m} M$ HEPES, pH 7.5 for yPGK and $20 \mathrm{~m} M$ HEPES, pH 7.5 for rPGK. Except where noted, optical densities at $280 \mathrm{~nm}$ of between 0.3 and 0.4 were used for all experiments. Samples were deoxygenated by flowing ultrapure argon through sealed cuvettes as first described by Englander et al. (30) and modified for use in RTP studies as previously described (21).

Phosphorescence. Time-resolved RTP data were collected using a setup similar to that described by Mersol et al. (8) (Fig. 1a). The second harmonic of a Spectra-Physics (Mountain View, CA) model DCR-11 Nd:YAG laser provided a 7-9 ns pulse at $532 \mathrm{~nm}$. This pulse pumped a dye laser (Quanta-Ray PDL-3) containing the dye rhodamine 590 that was tuned to lase at $560 \mathrm{~nm}$. The $560 \mathrm{~nm}$ light then passed through a second harmonic generating crystal (Inrad, Northvale, NJ) yielding the $280 \mathrm{~nm}$ pulse that excited the sample. The sample's emission at $450 \mathrm{~nm}$, the peak of Trp phosphorescence emission, was separated by an ISA (Edison, NJ) optical monochromator and detected by a Hamamatsu (Bridgewater, NJ) R3550 photomultiplier tube (PMT) operating in photon-counting mode. The signal from the PMT was sent into a Pacific Instruments (Concord, CA) model AD6 amplifier/discriminator, collected by an EG\&G Ortec (Oak Ridge, TN) ACEMCS multichannel scaler card and stored in a personal computer.

Transient absorption. The transient absorption apparatus is shown in Fig. 1b. The $280 \mathrm{~nm}$ pulse for exciting the sample from the ground state was produced as described for the phosphorescence setup. The probe beam was provided by a Spectra Physics (Mountain View, CA) model 166 line tunable argon ion laser. Typically, the laser was tuned to the $457.9 \mathrm{~nm}$ line near the peak of Trp triplet absorption. Additional spectral information was obtained by use of the other argon laser lines (as red as $514.5 \mathrm{~nm}$ ) and the $632.8 \mathrm{~nm}$ line from a Uniphase (San Jose, CA) helium neon (He-Ne) laser. The low divergence of the laser probe beam allowed the collection of data from small $(<10 \mu \mathrm{L})$ samples of proteins, permitted the detector to be positioned far from the sample so that scattered light from sample emission did not interfere with the measurements (31) and, because of low noise, made it easy to obtain time-resolved kinetics.

Prior to traversing the sample, approximately $50 \%$ of the probe beam was reflected to a reference silicon diode detector (Thor Labs, Newton, NJ) in order to provide a measure of the initial laser intensity. The excitation pulse and remaining probe beam were aligned co-linearly before passing through the sample. The transmission of the probe beam through the sample was detected by a second $\mathrm{Si}$ diode detector.

In contrast to time-resolved luminescence where photon counts are accumulated as a function of time, transient absorption always requires recording the difference between two large signals corresponding to transmission in the absence and presence of excitation. respectively. Hence, common mode noise limits the ultimate sensitivity as does the dynamic range of the combined system of detector and electronics. To reduce noise on the probe beam when supplied by the argon laser, the beam was passed through a Thor Labs (Newton, NJ) model CR200 laser intensity stabilizer with a bandwidth of $1 \mathrm{MHz}$ prior to probing the transient absorption. Neutral density filters placed after the laser intensity stabilizer insured that the argon beam power was $0.5 \mathrm{~mW}$ or less.

The detected signals from both Si diodes were sent to an Analogic (Peabody, MA) Data 6000 Universal Waveform Analyzer (D6000) 

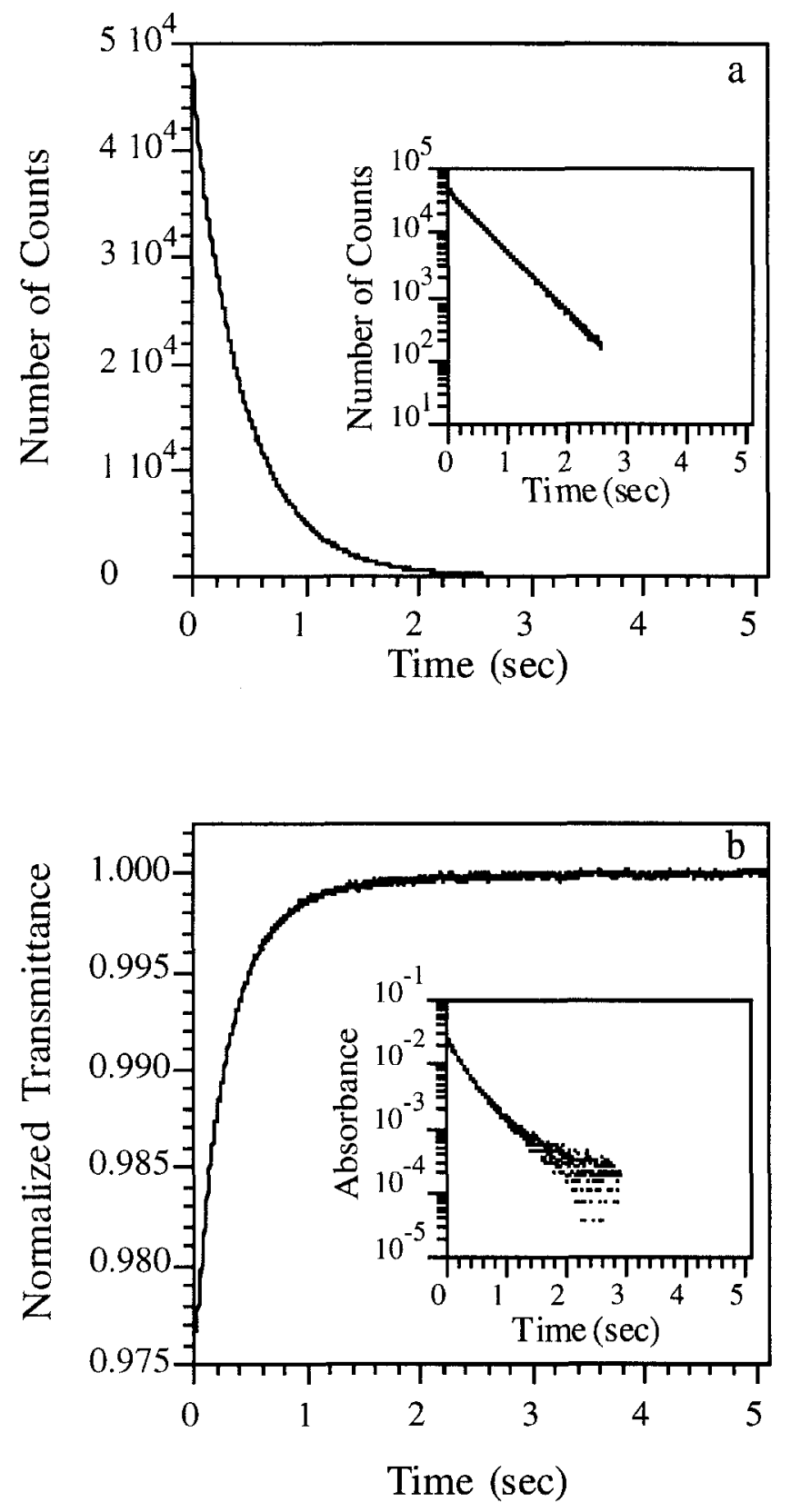

Figure 2. Room temperature phosphorescence (a) and transient transmission (b) of deoxygenated apoAz in $50 \mathrm{~m} M$ Tris, pH 7.5. The phosphorescence is the sum of $100 \mathrm{UV}$ laser shots and the transient absorption is the average of 100 shots. The insets display the data on a semilogarithmic scale. The semilogarithmic display for the transient transmission data was obtained by subtracting the normalized data from 1. Fitting parameters for the data are given in Table 1.

for digitization and analysis. When triggered by the Q-switch of the $\mathrm{Nd}: Y A G$, the D6000 collected transient absorption data at a sample interval determined by the length of the decay, where the minimum sample interval was $20 \mu \mathrm{s}$.

Data analysis. Phosphorescence data were fit to a sum of exponentials using the Marquadt $\chi^{2}$ method as implemented by the PTI (South Brunswick, NJ) program Fldec. For transient absorption, the transmission of the sample is given by the Beer-Lambert law:

$$
\frac{I_{s}}{I_{r}}=\frac{a}{1-a} 10^{-\left(\sum_{i} \epsilon_{i} \ell C_{i} \exp \left(-t / \tau_{i}\right)\right)}
$$

$I_{s}$ is the signal detected by the Si diode following the sample and $I_{r}$ is the signal from the reference detector. The fraction of the argon intensity that is incident on the sample is given by a and $(1-a)$ is the fraction of the intensity that is incident on the reference detector. $\ell$ is the path length, $1 \mathrm{~cm}$ for these experiments; $\epsilon_{\mathrm{i}}$ is the extinction coefficient of the ith-absorbing species and $C_{i}$ is the concentration of the ith species. For small optical densities, Eq. 1 may be approximated as:

$$
\frac{I_{S}}{I_{r}}=\frac{a}{1-a}\left[1-\sum_{i} A_{i} e^{\left(-1 / T_{i}\right)}\right]
$$

where $A_{i}$ is the amplitude of the contribution from the ith-absorbing species and $\tau_{\mathrm{i}}$ is the lifetime of the ith species. The transient absorption data were fit to Eq. 2 using the Marquadt method as implemented by the Jandel Scientific (San Rafael, CA) program TableCurve. The use of Eq. 2 rather than Eq. $\mathbf{I}$ is valid because all of the detected transient absorption signals displayed transmission changes of $5 \%$ or less, which correspond to an excited-state optical density of 0.02 or less.

The ability of TableCurve to fit the transient absorption data correctly despite the small magnitude of the signal was tested using data simulated by electronic modulation of the beam from a Uniphase (San Jose, CA) helium neon laser. The electronic modulation was provided by an RC circuit that drove the CR200 laser intensity stabilizer. TableCurve fits of these simulated data resulted in lifetimes within $8 \%$ of the $\mathrm{RC}$ time constant.

The small magnitude of the transient absorption signal led to obvious fluctuations in the data due to digitization (for example, see Fig. 4). The digitization also resulted in residuals (experimental data minus fit) that were not randomly distributed about zero as expected for a good fit to the data (data not shown). To ensure that neither the fluctuation or nonrandom residuals adversely affected the fits, data sets with known lifetimes and with and without simulated digitization fluctuations were calculated using Eq. 2. The presence of simulated digitization did not affect the fits to the calculated data sets.

Average lifetimes $\left(\tau_{\mathrm{av}}\right)$ were calculated according to:

$$
\tau_{\mathrm{av}}=\frac{\sum_{\mathrm{i}}\left(\mathrm{A}_{\mathrm{i}} \tau_{\mathrm{i}}\right)}{\sum_{\mathrm{i}} \mathrm{A}_{\mathrm{i}}} .
$$

\section{RESULTS}

\section{ApoAz}

Azurin is a monomeric, copper-containing protein with a single Trp residue in position $48(32,33)$. Both apoAz and azurin reconstituted with $\mathrm{Zn}$ replacing the copper atom display RTP $(2,13,34)$ with multiexponential decays (13), reflecting the presence of ground-state heterogeneity. Experiments on apoAz were conducted using highly concentrated solutions with optical densities at $280 \mathrm{~nm}$ of 1 or greater. The phosphorescence from these samples at $\mathrm{pH} 7.5$ showed a biexponential decay with lifetimes of hundreds of milliseconds (Fig. 2a, Table 1). The transient absorption from the same samples was also fit to a biexponential (Fig. 2b, Table 1). The average lifetimes of the phosphorescence and transient absorption for apoAz were within $10-30 \%$ of each other. The phosphorescence average lifetimes measured before and after transient absorption data were taken varied by $5 \%$. While the lifetimes measured by transient absorption and phosphorescence were similar for this protein, it is important to note that the relative contribution to the total transient absorption and phosphorescence of the two lifetimes differed significantly. The differences may reflect differences in transition oscillator strengths associated with the excitation of the different decay components. 
Table 1. Decay parameters for RTP and transient absorption data of apoAz in $50 \mathrm{~m} M$ Tris, $\mathrm{pH} 7.5^{*}$

\begin{tabular}{|c|c|c|c|c|c|c|}
\hline Experiment type & Amplitude & $\begin{array}{l}\text { Fractional } \\
\text { amplitude }\end{array}$ & $\begin{array}{l}\text { Lifetime } \\
\quad(\mathrm{ms})\end{array}$ & $\%$ intensity & $\begin{array}{l}\text { Average } \\
\text { lifetime } \\
\text { (ms) }\end{array}$ & $\begin{array}{c}\text { Normalized } \\
\chi^{2}\end{array}$ \\
\hline \multirow[t]{2}{*}{ Phosphorescence } & $4.61 \times 10^{4}$ & 0.92 & 460 & 96 & \multirow[t]{2}{*}{444} & \multirow[t]{2}{*}{1.03} \\
\hline & $3.85 \times 10^{3}$ & 0.08 & 251 & 4 & & \\
\hline \multirow[t]{2}{*}{ Transient absorption } & 0.0062 & 0.24 & 612 & 46 & \multirow[t]{2}{*}{317} & \multirow[t]{2}{*}{1.42} \\
\hline & 0.0197 & 0.76 & 225 & 54 & & \\
\hline
\end{tabular}

*Transient absorption fits are to Eq. 2. The data are displayed in Fig. 2.
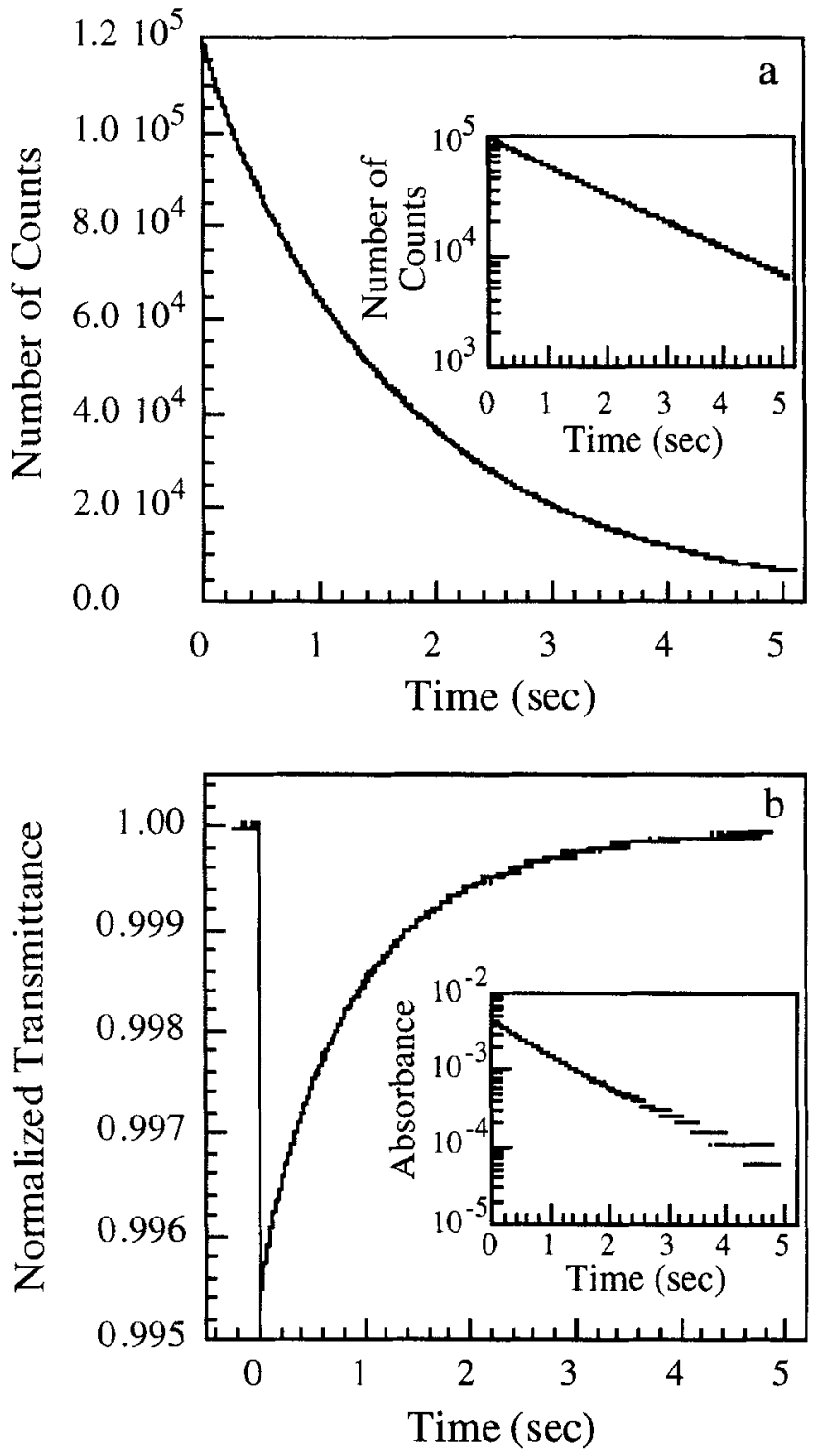

Figure 3. Room temperature phosphorescence (a) and transient transmission (b) of $6 \mu M$ deoxygenated AP in $50 \mathrm{~m} M$ Tris, $\mathrm{pH}$ 7.5. The phosphorescence is the sum over $300 \mathrm{UV}$ laser shots and the transient absorption is the average of 300 shots. The insets display the data on a semilogarithmic scale (as in Fig. 2). Fitting parameters for the data are given in Table 2.

\section{$\mathbf{A P}$}

Alkaline phosphatase is a dimer containing three Trp residues per subunit (35-37) whose RTP has been extensively studied $(5,8,38,39)$ and has been shown to arise from Trp $109(8,39)$. The RTP decay of AP in well-deoxygenated solutions is nearly monoexponential $(40,41)$ with a lifetime on the order of $2 \mathrm{~s}$ (Fig. 3a, Table 2), while its lifetime in aerated solutions is approximately $3 \mathrm{~ms}$. Due to the long triplet state lifetime of AP, we anticipated that on time scales of milliseconds and longer the absorption of the $457.9 \mathrm{~nm}$ line of the argon laser would be dominated by the triplet state because the peak of Trp triplet-triplet absorption occurs around $450 \mathrm{~nm}$ (Fig. 3b, Table 2) (42). This assumption was confirmed by experiments comparing the absorption at 457.9 $\mathrm{nm}, 514.5 \mathrm{~nm}$ and $632.8 \mathrm{~nm}$ of deoxygenated, photoexcited AP. The absorption of the $514.5 \mathrm{~nm}$ light was approximately one third that of the $457.9 \mathrm{~nm}$ laser line while the data at $632.8 \mathrm{~nm}$ showed no significant signal (data not shown).

Experiments performed using aerated solutions of AP demonstrated good agreement between the lifetimes determined by phosphorescence and those from transient absorption (Table 2). However, for deoxygenated AP samples, the ratio between $\tau_{\mathrm{av}}$ for transient absorption and $\tau_{\mathrm{av}}$ for phosphorescence varied from experiment to experiment, with some evidence that transient absorption lifetimes increased significantly with increasing RTP lifetimes. Assuming the transient absorption contains a component identical to the time-resolved phosphorescence, the transient absorption can be fit using the amplitudes and lifetimes from phosphorescence plus an additional component according to the form:

normalized transmittance

$$
=1-A\left(\frac{A_{1 p}}{A_{2 p}} e^{-t / \tau_{1}}+e^{-t / \tau_{2 p}}\right)-B e^{-t / \tau}
$$

where $A_{1 p}$ and $A_{2 p}$ are the amplitudes from the fit to the phosphorescence decay and $\tau_{1 p}$ and $\tau_{2 p}$ are the phosphorescence lifetimes. A, B and $\tau$ are adjustable parameters. Without the additional exponential term the transient absorption data could not be fit by this equation.

The goodness of fit was comparable to that for the unconstrained results. The value of $\tau$ in Eq. 4 ranged from 50 to $700 \mathrm{~ms}$, and this component contributed up to $50 \%$ of the transient absorption signal. The additional lifetime for the transient absorption presumably results from a dark (nonemitting) state such as the Trip species or perhaps from a dark triplet state associated with one of the two other Trp residues in AP. Despite its oxygen sensitivity, this contri- 
Table 2. Decay parameters for RTP and transient absorption data of $6 \mu M$ AP in $50 \mathrm{~m} M$ Tris, pH $7.5^{*}$

\begin{tabular}{|c|c|c|c|c|c|c|}
\hline & Amplitude & $\begin{array}{l}\text { Fractional } \\
\text { amplitude }\end{array}$ & $\begin{array}{l}\text { Lifetime } \\
\text { (s) }\end{array}$ & $\%$ intensity & $\begin{array}{l}\text { Average lifetime } \\
\text { (s) }\end{array}$ & $\begin{array}{c}\text { Normalized } \\
\chi^{2}\end{array}$ \\
\hline Phosphorescencet & $6.62 \times 10^{2}$ & 1.0 & 0.00258 & 100 & $2.58 \times 10^{-3}$ & 2.68 \\
\hline Transient absorption $\dagger$ & $1.7 \times 10^{-3}$ & 1.0 & 0.0029 & 100 & $2.9 \times 10^{-3}$ & 1.12 \\
\hline \multirow{2}{*}{ Phosphorescence $¥$} & $1.31 \times 10^{5}$ & 0.96 & 1.79 & 99 & 1.74 & 1.12 \\
\hline & $5.52 \times 10^{3}$ & 0.04 & 0.47 & 1 & & \\
\hline \multirow[t]{5}{*}{ Transient absorption $\ddagger$} & $2.33 \times 10^{-3}$ & 0.52 & 1.33 & 70 & 0.98 & 1.16 \\
\hline & $2.16 \times 10^{-3}$ & 0.48 & 0.60 & 30 & & \\
\hline & $1.04 \times 10^{-3}$ & 0.233 & $1.79 \S$ & 41.5 & 1.00 & 10.28 \\
\hline & $5.91 \times 10^{-5}$ & 0.013 & $0.47 \S$ & 0.6 & & \\
\hline & $3.36 \times 10^{-3}$ & 0.754 & 0.77 & 57.9 & & \\
\hline
\end{tabular}

*Transient absorption fits are to Eq. 2 except as indicated. The data for the deoxygenated sample are displayed in Fig. 3.

†Aerated samples.

†Deoxygenated samples

§These lifetimes were held fixed according to Eq. 4.

bution is not likely to arise from $\mathrm{e}_{\mathrm{aq}}^{-}$because there is no transient absorption signal at $632.8 \mathrm{~nm}$ for deoxygenated AP samples.

Given the long lifetime of AP, a possible experimental source of discrepancy between the phosphorescence and transient absorption data is the diffusion of excited molecules out of the small region probed in the transient absorption experiments. While estimates of the magnitude of this effect indicated it was not likely significant, the possibility was tested by enlarging the transverse size of the interaction region 3.5 times, leading to greater than an order of magnitude increase in the diffusion time across the probed region for an AP molecule. As expected the data show no detectable effect on the transient absorption decay.

Some of the discrepancies between lifetimes determined from RTP and transient absorption data may arise from the difficulty in fitting the latter data due to the small dynamic range. For example, two transient absorption experiments on the same deoxygenated AP sample had average lifetimes of 1.1 and $1.2 \mathrm{~s}$ while the long lifetime of the unconstrained



Figure 4. Transient absorption of $14 \mu M$ yPGK in $20 \mathrm{~m} M$ HEPES, $\mathrm{pH} 7.5$ at $1^{\circ} \mathrm{C}$ in aerated and deoxygenated solution. Data are the average of $300 \mathrm{UV}$ laser pulses. two-component fits were 1.7 and $3.0 \mathrm{~s}$, respectively. Constraining the $1.7 \mathrm{~s}$ fit to $3.0 \mathrm{~s}$ changed the adequacy of the fit, as measured by $r^{2}$, by only $0.05 \%$ indicating the difficulty in determining individual lifetimes and amplitudes from the transient absorption data.

\section{PGK}

Yeast PGK is a monomeric enzyme containing 2 Trp residues $(43,44)$, whose phosphorescence has been reported at 1,20 and $25^{\circ} \mathrm{C}$ at $\mathrm{pH} 7.5$ with lifetimes on the order of 100 ms (the actual values depend on the temperature and the buffer used) $(4,28,29)$. We measured the phosphorescence of yPGK obtained from Sigma and from Boehringer Mannheim both at $1^{\circ} \mathrm{C}$ and at room temperature in triethanolamine as well as in HEPES at pH 7.5. In these experiments, the long-lived emission was extremely weak, contributing only $1 \%$ of the phosphorescence signal. Interestingly, yPGK in triethanolamine exhibited an oxygen-sensitive transient absorption at $1^{\circ} \mathrm{C}$, and in HEPES this protein showed an oxygen-sensitive transient absorption at both $1^{\circ} \mathrm{C}$ (Fig. 4) and room temperature. The transient absorption signal for yPGK even in deoxygenated HEPES solutions corresponded to a less than $0.1 \%$ change in transmission relative to the baseline making fits to these data unreliable.

Rabbit muscle PGK is a monomeric enzyme containing four Trp residues (43) but shows no long-lived phosphorescence, presumably because all the Trp are near the surface and hence in a flexible environment. As in the case of yPGK, the transient absorption of rPGK at $457.9 \mathrm{~nm}$ did reveal a long-lived component (Fig. 5, Table 3). This transient was virtually indistinguishable in aerated and deoxygenated solution (Fig. 5) and the amplitude of the long-lived transient increased upon changing the probe wavelength from 457.9 to $514.5 \mathrm{~nm}$ (Fig. 5b). These data suggest that the long-lived transient absorption of rabbit PGK arises from a state other than the Trp triplet state.

As in the case of yPGK, the transient absorption signal from rPGK exhibited an oxygen-sensitive component. However, the contribution of this component to the overall transient absorption signal intensity was only $1-2 \%$. This small component was also present in the deoxygenated $514.5 \mathrm{~nm}$ absorption data though not detectable at $632.8 \mathrm{~nm}$. Hence, 

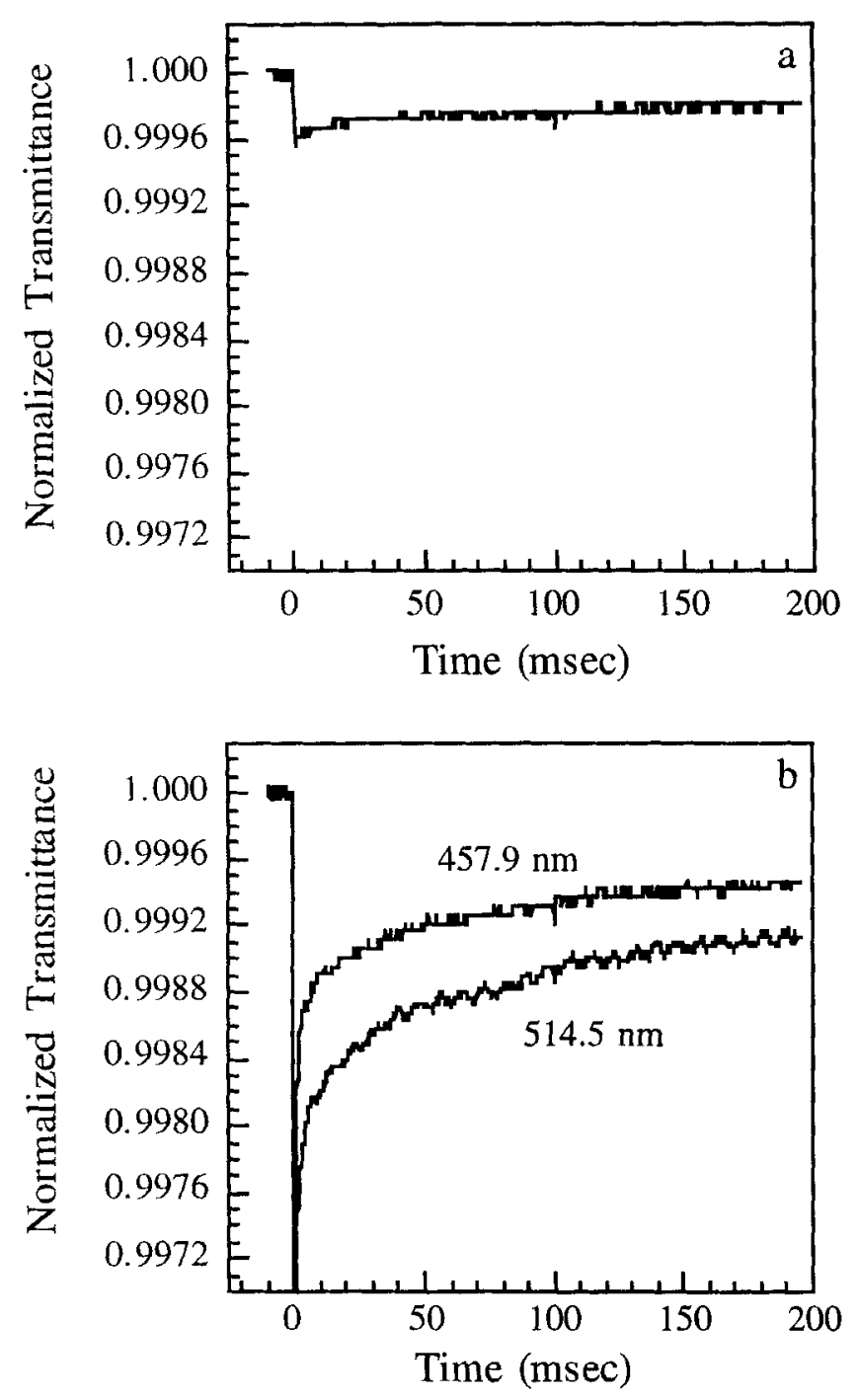

Figure 5. Room temperature transient absorption of $15 \mu M \mathrm{rPGK}$ in $20 \mathrm{~m} M$ HEPES, pH 7.5. Displayed are the persistence of the long component in aerated solution at $457.9 \mathrm{~nm}$ (a) and the increase in signal upon changing the probe wavelength from $457.9 \mathrm{~nm}$ to 514.5 for the deoxygenated sample (b). Transient absorption data are the average of 300 UV laser pulses. Lifetimes and amplitudes for the deoxygenated data are given in Table 3 .

this short component is tentatively assigned to the Trp triplet state.

\section{DISCUSSION}

Based on the rapid developments of Trp RTP spectroscopy, it is becoming increasingly clear that measurement of the Trp triplet state lifetime can provide new insight into conformational changes and protein dynamics. This is a direct consequence of the forbidden nature of the optical transition to the ground state that leads to a long intrinsic lifetime and hence to an enhanced sensitivity to competitive quenching mechanisms relative to the short-lived singlet state. The long radiative lifetime (11.5 s) (45), however, implies a small triplet state quantum yield in the presence of strong quenching. Hence, the study of alternative methods to measure the triplet state dynamics is important. The present study ex-
Table 3. Decay parameters for three exponential fits to the transient absorption of deoxygenated $15 \mu M$ rabbit muscle PGK in 20 $\mathrm{m} M$ HEPES, $\mathrm{pH} 7.5$ at room temperature*

\begin{tabular}{ccccccc}
\hline $\begin{array}{c}\text { Probe } \\
\text { wave- } \\
\text { length } \\
(\mathrm{nm})\end{array}$ & $\begin{array}{c}\text { Amplitude } \\
\left(\times 10^{-3}\right)\end{array}$ & $\begin{array}{c}\text { Frac- } \\
\text { tional } \\
\text { ampli- } \\
\text { tude }\end{array}$ & $\begin{array}{c}\text { Lifetime } \\
(\mathrm{ms})\end{array}$ & $\begin{array}{c}\% \\
\text { intensity }\end{array}$ & $\begin{array}{c}\text { Average } \\
\text { lifetime } \\
(\mathrm{ms})\end{array}$ & $\begin{array}{c}\text { Normal- } \\
\text { ized } \\
\chi^{2}\end{array}$ \\
\hline \multirow{2}{*}{457.9} & 0.834 & 0.15 & 440 & 96 & 70 & 1.59 \\
& 0.519 & 0.10 & 23 & 3 & & \\
514.5 & 4.08 & 0.75 & 0.6 & 1 & & \\
& 1.40 & 0.39 & 373 & 96.9 & 151 & 2.91 \\
& 0.679 & 0.19 & 21 & 2.7 & & \\
\hline
\end{tabular}

*Fits are to Eq. 2 and the data are displayed in Fig. 5.

amines the effectiveness of monitoring the transient absorption that results following the population of the triplet state by intersystem crossing from the originally excited singlet state. While this technique is intrinsically more challenging, for reasons discussed below, the data clearly show that new information is contained in such measurements.

Early measurements of the triplet state lifetime in solution were in fact based on transient absorption because of the low phosphorescence quantum yield. Lifetimes of the various indole derivatives varied between 11 and $29 \mu \mathrm{s}$ $(22,23,46-48)$ leading to the conclusion that the highly flexible environment of the free indole leads to strong quenching of the triplet state lifetime. However, more recent measurements based on time-resolved phosphorescence of indole in fluid solutions demonstrated that these earlier measurements may not reflect the intrinsic lifetime of free indole but rather a lifetime shortened by collisional quenching by ground-state indole molecules. While there was agreement between the two types of measurements at high indole concentrations, the phosphorescence lifetime increased to approximately 1 ms when the indole concentration was reduced from the millimolar to the micromolar level (7).

Such collisional quenching is expected to be reduced when the Trp is buried inside a protein, and hence it is not surprising that reasonable agreement between transient absorption and time-resolved phosphorescence is reported in experiments on cod parvalbumin $(24,25)$. Equally significant is that our measurements on the single Trp-containing apoAz show reasonable agreement between transient absorption and time-resolved phosphorescence, even though these lifetimes are much longer, leading to the possibility of competing reactions and production of photoproducts that would lead to differences in the results.

Indeed, our results for AP, also characterized by a longlived Trp triplet state, demonstrate that such competitive processes can lead to differences in the measured lifetimes in proteins. Average decay rates calculated (using Eq. 3) from fits to the AP transient absorption data differ considerably from those extracted from phosphorescence decays. When the sample contains residual oxygen, a lower phosphorescence average lifetime is obtained, and in these cases the transient absorption $\tau_{\mathrm{av}}$ appears to be lower than the phosphorescence $\tau_{a v}$. When the RTP $\tau_{a v}$ is long, indicating better deoxygenation, the transient absorption $\tau_{\mathrm{av}}$ is sometimes lon- 
ger than the RTP $\tau_{\mathrm{av}}$. The importance of deoxygenation is also indicated by the good agreement between RTP and transient absorption for aerated samples of AP.

The longest component of the yPGK RTP had a lifetime on the order of $100 \mathrm{~ms}$, but it contributed less than $1 \%$ of the RTP signal. This contrasts sharply with the AP and apoAz RTP data where, in both cases, over $95 \%$ of the signal is contributed by the long-lived component. The lack of a significant long-lived phosphorescence signal from yPGK is also unexpected in view of the published RTP data $(4,28,29)$ and the oxygen-sensitive transient absorption of yPGK. Hence, the small long-lived yPGK RTP signal could be due to contaminants or to some unidentified change in the environment of Trp 333, the phosphorescing Trp in yPGK, in our measurements.

The two Trp residues in yeast PGK are evolutionarily conserved $(49,50)$; therefore, we expected to see a long-lived oxygen-sensitive lifetime in rPGK. However, the oxygensensitive component of the rPGK transient absorption has a lifetime that is only on the order of $1 \mathrm{~ms}$. Yeast PGK also shows a short oxygen-sensitive transient absorption component, and it is likely that this component arises from the same Trp residue in both proteins.

The long-lived rPGK transient cannot arise directly from either the Trp triplet state or $\mathbf{e}_{a q}^{-}$because it is present with an apparently unmodified lifetime in both deoxygenated and aerated solutions. It must, therefore, arise from either the Trp neutral or cation radical. The peak of the neutral radical absorption occurs at $510 \mathrm{~nm}$ with an $€$ of $1750 \mathrm{M}^{-1} \mathrm{~cm}^{-1}$ (26) while the cation radical peak absorption is at $570 \mathrm{~nm}$ with an $\epsilon$ of $2600 \mathrm{M}^{-1} \mathrm{~cm}^{-1}$ (26). Due to the difference in extinction coefficient, the doubling of the transient absorption signal between $457.9 \mathrm{~nm}$ and $514.5 \mathrm{~nm}$ is not sufficient to distinguish between the two species. Rabbit PGK has two Trp residues that are not present in yPGK, it is possible that the rPGK long-lived transient arises from one of these additional Trp. Hence, in order to definitively assign the longlived rPGK transient absorption, mutation of the Trp residues is necessary.

While it is clear that new information is available from transient absorption, we note that the application of this approach to weakly absorbing systems, while allowing measurement of the lifetimes of the individual components, is difficult due to the small change in transmitted optical radiation. In practice, the maximum induced absorption change in these systems is a few percent. Using a 14 bit digitizer with adequate time resolution, a single transient response produces three decades or less of dynamic range, compared to time-resolved luminescence signals that easily produce lifetime data extending over four decades or more (see insets to Figs. 2 and 3). This lack of dynamic range makes it difficult to resolve different contributions to the decay and results in relatively large uncertainties in the parameters obtained by curve fitting. Higher protein concentrations as well as stronger excitation beams can increase the transient $a b-$ sorption signal but high protein concentrations are sometimes hard to obtain and/or may lead to protein association and modification, while higher excitation energy may result in photochemical complications.

The information obtained from protein transient absorption experiments can complement the Trp triplet state data obtained from RTP. The presence of additional components in the transient absorption data indicates the production of dark states that cannot be probed by phosphorescence. Although it is difficult to obtain individual lifetimes from transient absorption data, $\tau_{\mathrm{av}}$ may be calculated and compared to results from RTP.

The presence of additional decays in the transient absorption data may provide insight into mechanisms of tripletstate quenching. Definitive species identification requires comparison of aerated and deoxygenated solutions as well as comparison of transient absorption at various wavelengths. Assignment of the transiently absorbing species to particular Trp residues is complicated in multi-tryptophan proteins and may require the use of single-tryptophan mutants. The identification of specific transiently absorbing species in different proteins should lead to a better understanding of triplet-state photochemistry and the affects of local environment on $\operatorname{Trp}$ residues.

Acknowledgements-The authors thank Dr. Joseph Schauerte for assistance with phosphorescence measurements and helpful discussions and Dr. Bruce Schlyer for helpful discussions and experimental suggestions. This work was supported by the National Institute on Aging grant NIA AG09761. Anne Gershenson was supported by an NIH Molecular Biophysics Training Grant (GM08270-05).

\section{REFERENCES}

1. Saviotti, M. L. and W. C. Galley (1974) Room temperature phosphorescence and the dynamic aspects of protein structure. Proc. Natl. Acad. Sci. USA 71, 4154-4158.

2. Vanderkooi, J. M., D. B. Calhoun and S. W. Englander (1987) On the prevalence of room-temperature protein phosphorescence. Science 236, 568-569.

3. Vanderkooi, J. M. (1992) Tryptophan phosphorescence from proteins at room temperature. In Topics in Fluorescence Spectroscopy, Vol. 3: Biochemical Applications (Edited by J. R. Lakowicz), pp. 113-136. Plenum Press, New York.

4. Gonnelli, M. and G. B. Strambini (1995) Phosphorescence lifetime of tryptophan in proteins. Biochemistry 34, 13847-13857.

5. Papp, S. and J. M. Vanderkooi (1989) Tryptophan phosphorescence at room temperature as a tool to study protein structure and dynamics. Photochem. Photobiol. 49, 775-784.

6. Strambini, G. B. and M. Gonnelli (1985) The indole nucleus triplet-state lifetime and its dependence on solvent microviscosity. Chem. Phys. Lett. 115, 196-200.

7. Strambini, G. B. and M. Gonnelli (1995) Tryptophan phosphorescence in fluid solution. J. Am. Chem. Soc. 117, 7646-7651.

8. Mersol, J. V., D. G. Steel and A. Gafni (1991) Quenching of tryptophan phosphorescence in Escherichia coli alkaline phosphatase by long-range transfer mechanisms to external agents in the rapid-diffusion limit. Biochemistry 30,668-675.

9. Schlyer, B. D., D. G. Steel and A. Gafni (1995) Direct kinetic evidence for triplet state energy transfer from Escherichia coli alkaline phosphatase tryptophan 109 to bound terbium. J. Biol. Chem. 270, 22890-22894.

10. Mersol, J. V., D. G. Steel and A. Gafni (1993) Detection of intermediate protein conformations by room temperature tryptophan phosphorescence spectroscopy during denaturation of Escherichia coli alkaline phosphatase. Biophys. Chem. 48, 281291.

11. Subramaniam, V., N. C. Bergenhem, A. Gafni and D. G. Steel (1995) Phosphorescence reveals a continued slow annealing of the protein core following reactivation of Escherichia coli alkaline phosphatase. Biochemistry 34, 1133-1136.

12. Gonnelli, M., A. Puntoni and G. B. Strambini (1992) Tryptophan phosphorescence of ribonuclease $\mathrm{T} 1$ as a probe of protein flexibility. J. Fluoresc. 2, 157-165.

13. Hansen, J. E., D. G. Steel and A. Gafni (1996) Detection of a 
pH-dependent conformational change in azurin by time-resolved phosphorescence. Biophys. J. 71, 2138-2143.

14. Schauerte, J., D. G. Steel and A. Gafni (1992) Demonstration of time resolved circularly polarized phosphorescence and application to study of protein-ligand binding. Proc. Natl. Acad. Sci. USA 89, 10154-10158.

15. Schauerte, J. A., A. Gafni and D. G. Steel (1996) Improved differentiation between luminescence decay components by use of time-resolved optical activity measurements and selective lifetime modulation. Biophys. J. 70, 1996-2000.

16. Strambini, G. B., P. Cioni, A. Peracchi and A. Mozzarelli (1992) Conformational changes and subunit communication in tryptophan synthase: effect of substrates and substrate analogs. Biochemistry 31, 7535-7542.

17. Bergenhem, N. C., B. D. Schlyer, D. G. Steel, A. Gafni, U. Carlsson and B. H. Jonsson (1994) Lack of correspondence between the room-temperature phosphorescence decay-components and $\operatorname{Trp}$ residues in a series of $\operatorname{Trp} \rightarrow$ Cys or $\operatorname{Trp} \rightarrow$ Phe mutants of human carbonic anhydrase II. FEBS Lett. 353, 177179.

18. Strambini, G. B., P. Cioni and P. F. Cook (1996) Tryptophan luminescence as a probe of enzyme conformation along the $O$ acetylserine sulfhydrylase reaction pathway. Biochemistry 35 , $8392-8400$

19. Gabellieri, E., S. Rahuel-Clermont, G. Branlant and G. B. Strambini (1996) Effects of NAD+-binding on the luminescence of tryptophans 84 and 310 of glyceraldehyde-3-phosphate dehydrogenase from Bacillus stearothermophilus. Biochemistry $35,12549-12559$.

20. Subramaniam, V., A. Gafni and D. G. Steel (1996) Time-resolved tryptophan phosphorescence spectroscopy: a sensitive probe of protein folding and structure. IEEE J. Quant. Elec. 2, $1107-1114$.

21. Schauerte, J., D. Steel and A. Gafni (1997) Time resolved room temperature phosphorescence in protein studies. Methods Enzymol. 278, 49-71.

22. Grossweiner, L. I. and Y. Usui (1971) Flash photolysis and inactivation of aqueous lysozyme. Photochem. Photobiol. 13, $195-214$

23. Ghiron, C., M. Bazin and R. J. Santus (1988) Characterization of the indole triplet excited state in proteins utilizing laser flash photolysis. J. Biochem. Biophys. Methods 15, 337-348.

24. Sudhakar, K., C. M. Phillips, S. A. Williams and J. M. Vanderkooi (1993) Excited states of tryptophan in cod parvalbumin: identification of a short-lived emitting triplet state at room temperature. Biophys. J. 64, 1503-1511.

25. Sudhakar, K., C. M. Phillips, C. S. Owen and J. M. Vanderkooi (1995) Dynamics of parvalbumin studied by fluorescence emission and triplet absorption spectroscopy of tryptophan. Biochemistry 34, 1355-1363.

26. Redpath, J. L., R. Santus, J. Ovadia and L. I. Grossweiner (1975) The oxidation of tryptophan by radical anions. Int. J. Radiat. Biol. 27, 201-204.

27. Grossweiner, L. I., A. G. Kaluskar and J. F. Baugher (1976) Flash photolysis of enzymes. Int. J. Radiat. Biol. 29, 1-16.

28. Cioni, P., A. Puntoni and G. B. Strambini (1993) Tryptophan phosphorescence as a monitor of the solution structure of phosphoglycerate kinase from yeast. Biophys. Chem. 46, 47-55.

29. Cioni, P. and G. B. Strambini (1994) Pressure effects on protein flexibility monomeric proteins. J. Mol. Biol. 242, 291-301.

30. Englander, S. W., D. B. Calhoun and J. J. Englander (1987) Biochemistry without oxygen. Anal. Biochem. 161, 300-306.

31. Chan, S. S. and R. H. Austin. 1984. Laser photolysis in bio- chemistry. In Methods of Biochemical Analysis, Vol. 30 (Edited by D. Glick), pp.105-139. John Wiley \& Sons, New York.

32. Adman, E. T. and L. H. Jensen (1981) Structural features of azurin at $2.7 \AA$ resolution. Isr. J. Chem. 21, 8-12.

33. Nar, H., A. Messerschmidt, R. Huber, M. van de Kamp and C. Gerard (1991) Crystal structure analysis of oxidized Pseudomonas aeruginosa azurin at $\mathrm{pH} 5.5$ and $\mathrm{pH}$ 9.0. J. Med. Biol. 221, 765-772.

34. Strambini, G. B. and E. Gabellieri (1991) Phosphorescence from trp-48 in azurin: influence of $\mathrm{Cu}(\mathrm{II}), \mathrm{Cu}(\mathrm{I}), \mathrm{Ag}(\mathrm{I})$, and $\mathrm{Cd}(\mathrm{II})$ at the coordination site. J. Phys. Chem. 95, 4352 4356.

35. Wyckoff, H. W., M. Handschumacher, H. M. K. Murthy and J. M. Sowadski (1983) The three dimensional structure of alkaline phosphatase from E. coli. Adv. Enzymol. 55, 453-480.

36. Sowadski, J. M., M. D. Handschumacher, H. M. K. Murthy, B. A. Foster and H. W. Wyckoff (1985) Refined structure of alkaline phosphatase from Escherichia coli at $2.8 \AA$ resolution. $J$. Mol. Biol. 186, 417-433.

37. Kim, E. E. and H. W. Wyckoff (1989) Structure of alkaline phosphatases. Clin. Chim. Acta 186, 175-188.

38. Mersol, J. V. (1992) Room temperature tryptophan phosphorescence spectroscopy as a probe of protein structure and dynamics. Ph.D. thesis, University of Michigan, Ann Arbor.

39. Strambini, G. B. (1987) Quenching of alkaline phosphatase phosphorescence by $\mathrm{O}_{2}$ and $\mathrm{NO}$ : evidence for inflexible regions of protein structure. Biophys. J. 52, 23-28.

40. Schlyer, B. D., J. A. Schauerte, D. G. Steel and A. Gafni (1994) Time-resolved room temperature protein phosphorescence: nonexponential decay from single emitting tryptophans. Biophys. $J$. 67, 1192-1202.

41. Schlyer, B. D., J. A. Schauerte, D. G. Steel and A. Gafni (1994) The nonexponential decay of room temperature phosphorescence: evidence for several slowly interconverting or static protein conformers. In Time-Resolved Laser Spectroscopy in Biochemistry IV, (Edited by J. R. Lakowic) pp. 146-153. SPIE, Los Angeles.

42. Bent, D. V. and E. Hayon (1975) Excited state chemistry of aromatic amino acids and related peptides. III. Tryptophan. $J$. Am. Chem. Soc. 97, 2612-2619.

43. Fifis, T. and R. K. Scopes (1978) Purification of 3-phosphoglycerate kinase from diverse sources by affinity elution chromatography. Biochem. J. 175, 311-319.

44. Watson, H. C., N. P. C. Walker, P. J. Shaw, T. N. Bryant, P. L. Wendell, L. A. Fothergill, R. E. Perkins, S. C. Conroy, M. J. Dobson, M. F. Tuite, A. J. Kingsman and S. M. Kingsman (1982) Sequence and structure of yeast phosphoglycerate kinase. EMBO J. 1, 1635-1640.

45. Galley, W. C. and L. Stryer (1969) Triplet-singlet energy transfer in proteins. Biochemistry 8, 1831-1838.

46. Bent, D. V. and E. Hayon (1975) Excited state chemistry of aromatic amino acids and related peptides. I. Tyrosine. J. Am. Chem. Soc. 97, 2599-2606.

47. Volkert, W. A., R. R. Kuntz, C. A. Ghiron, R. F. Evans, R Santus and M. Baazin (1977) Flash photolysis of tryptophan and $\mathrm{N}$-acetyl-L-tryptophanamide; the effect of bromide on transient yields. Photochem. Photobiol, 26, 3-9.

48. Pepmiller, C., E. Bedwell, R. R. Kuntz and C. A. Ghiron (1983) A flash photolysis study of 1-methylindole. Photochem. Photobiol. 38, 273-280.

49. Mori, N., J. Singer-Sam and A. D. Riggs (1986) Evolutionary conservation of the substrate-binding cleft of phosphoglycerate kinases. FEBS Lett. 204, 313-317.

50. Watson, H. C. and J. A. Littlechild (1990) Isoenzymes of phosphoglycerate kinase: evolutionary conservation of the structure of this glycolytic enzyme. Biochem. Soc. Trans. 18, 187-190. 